\title{
O estado do Rio de Janeiro como limite sul e norte de distribuição de algumas espécies de aves
}

\author{
Francisco Mallet-Rodrigues
}

Laboratório de Ornitologia, Departamento de Zoologia, Instituto de Biologia, Universidade Fedral do Rio de Janeiro (UFRJ), 21944-970, Rio de Janeiro, RJ, Brasil. (fmallet@bol.com.br)

\begin{abstract}
The state of Rio de Janeiro as the southern and northern boundary of the distribution of some bird species. The state of Rio de Janeiro is located in a region that can be considered biogeographically important for harboring extreme geographical limits of the Serra do Mar and lowland forests, which are very significant for the distribution of several bird species. In this study, through extensive bibliographic search, 59 bird species were found with their southern ( 40 species) or northern ( 19 species) limit of distribution located in the state of Rio de Janeiro. Birds with the southern limit of distribution in Rio de Janeiro have been recorded in almost the entire state, except in the upper valley of the Paraíba do Sul river and the western portion of the coastal zone of the state. This birds species are predominantly associated with lowland and submontane forests and more than half is included in the list of species threatened with extinction in the state. Birds with the northern limit of distribution in the state of Rio de Janeiro can be classified into two basic groups according to their distribution pattern in the region. The first group consists of endemic montane Atlantic forest birds, while the second group consists of occasional visitors associated with aquatic environments. The low proportion of threatened bird species among those with the northern limit of distribution in Rio de Janeiro can be explained by the high proportion of species inhabiting montane forests, which are more protected than the lowland forests.
\end{abstract}

KEYWORDS. Atlantic Forest, Biogeography, geographical distribution, Serra do Mar, southeastern Brazil.

RESUMO. A região onde se localiza o estado do Rio de Janeiro pode ser considerada biogeograficamente importante por abrigar os limites geográficos extremos da Serra do Mar e das florestas dos tabuleiros (ou de baixada), formações bastante significativas para a distribuição de diversas espécies de aves. Nesse estudo, através de extensa consulta bibliográfica, foram encontradas 59 espécies de aves com seu limite sul (40 espécies) ou norte (19 espécies) de distribuição geográfica localizado no estado do Rio de Janeiro. As espécies com limite sul de distribuição no estado têm registros em quase todo o território fluminense, exceto na região do alto vale do rio Paraíba do Sul e na porção mais ocidental da costa fluminense. São espécies predominantemente associadas às florestas de baixada e submontanas e mais da metade está incluída na lista de espécies ameaçadas de extinção do estado. As espécies com limite norte de distribuição no Rio de Janeiro podem ser classificadas em dois grupos básicos quanto ao seu padrão de distribuição em território fluminense. O primeiro grupo é composto por espécies de ambientes florestais montanos e endêmicas da Mata Atlântica, enquanto o segundo grupo é constituído por visitantes ocasionais associados a ambientes aquáticos. A baixa representatividade de espécies ameaçadas entre aquelas com limite norte de distribuição no Rio de Janeiro pode ser explicada pela elevada proporção, entre suas espécies florestais, de representantes de matas serranas, melhor preservadas que as matas de baixada.

PALAVRAS-CHAVE. Mata Atlântica, Biogeografia, distribuição geográfica, serra do mar, sudeste do Brasil.

Diversos fatores abióticos e bióticos podem influenciar em diferentes níveis a distribuição das espécies biológicas (TERBORGH, 1971; MACARTHUR, 1972; Terborgh \& Weske, 1975). Os limites geográficos da distribuição de uma espécie, bem como sua abundância populacional, podem ser bastante dinâmicos ao acompanharem as mudanças (sazonais ou não) sofridas pelos fatores ambientais, embora seja difícil identificar os mecanismos através dos quais esses fatores afetam as populações (BRown \& Lomolino, 2006).

A intensa fragmentação florestal nas regiões tropicais altera significativamente a distribuição de muitas espécies, sendo, atualmente, uma das principais ameaças à biodiversidade mundial (DEAN, 1996). No leste do Brasil, na região da Floresta Atlântica, diversas espécies de aves endêmicas têm desaparecido localmente pela perda de habitats (WILLIS, 1979; AleIXo \& Vielliard, 1995; Mallet-Rodrigues et al., 2007), afetando de forma significativa, direta e indiretamente, os padrões locais de distribuição geográfica de inúmeras populações.

A considerável proporção de espécies endêmicas da Floresta Atlântica (aproximadamente 214 espécies entre as aves, segundo PARKER et al., 1996), torna esse bioma de extrema importância para a conservação da biodiversidade. Estudos incluindo variados táxons de animais e plantas têm reconhecido dois distintos centros de endemismo - norte e sul - nos domínios da Floresta Atlântica (Costa et al., 2000; Sigrist \& Carvalho, 2008), estando a área de separação entre esses centros de endemismo localizada na região dos estados do Espírito Santo e Rio de Janeiro. Também têm sido propostos quatro centros de endemismo - Pernambuco, Bahia Central, Bahia Costeira e Serra do Mar - para as aves passeriformes no bioma florestal atlântico (Silva et al., 2004).

O estado do Rio de Janeiro insere-se numa região ecologicamente importante do litoral brasileiro por abrigar os extremos geográficos de duas formações significativas para a distribuição de diversas espécies de aves - Serra do Mar e Floresta Pluvial dos Tabuleiros (Sick, 1997). A Serra do Mar é uma formação montanhosa, com cerca de $1000 \mathrm{~km}$ de extensão, que se estende ao longo da costa leste brasileira, desde o norte de Santa Catarina até a região centro-oriental do Rio de Janeiro, tendo seu limite norte na Serra do Desengano. É considerada um importante centro de endemismo para aves (Cracraft, 1985; Silva et al., 2004). A Floresta Pluvial dos Tabuleiros, também denominada "floresta costeira" ou "floresta litorânea", é uma formação 
florestal do domínio Atlântico, que ocupa tabuleiros costeiros, de origem terciária, em altitudes inferiores a 200 m, e que se distribui entre Pernambuco e o Rio de Janeiro (RizzinI, 1997). Fauna e flora das florestas dos tabuleiros são bastante relacionadas àquelas das florestas amazônicas (SICK, 1997; Rızzinı, 1997).

O objetivo básico desse estudo é apresentar uma breve discussão inicial sobre os padrões de distribuição das espécies de aves em seus limites geográficos extremos, tema ainda inexplorado na ornitologia brasileira. Para isso, serão investigados os possíveis padrões de distribuição local do conjunto de espécies de aves que apresentam seus limites sul e norte de ocorrência no estado do Rio de Janeiro.

\section{MATERIAL E MÉTODOS}

Foram considerados nesse estudo todas as espécies de aves que apresentam seus limites sul e norte de distribuição geográfica dentro da área do estado do Rio de Janeiro, segundo a literatura referente à avifauna brasileira (PINTO, 1938, 1944, 1978; SICK, 1997). Foram consultadas as listas de espécies de aves dos vizinhos estados de São Paulo e Espírito Santo, para uma melhor definição dos limites de distribuição de algumas espécies (WiLlis \& ONIKI, 2003; Silveira \& Uezu, 2011). Não foram incluídas, entretanto, as aves marinhas, as espécies migrantes neárticas, os invasores naturais em recente expansão de distribuição, bem como aquelas espécies com hipotético registro no estado, baseado em espécimes antigos e de origem questionável (PACHeCo \& Parrini, 1998a,b). A taxonomia e a sequência sistemática das espécies de aves seguem CBRO (2011). O habitat básico de cada espécie foi determinado através de consulta a PARKER et al. (1996) e Sick (1997). As espécies endêmicas da Mata Atlântica foram consideradas a partir de BENCKE et al. (2006). Para a determinação das espécies ameaçadas de extinção no estado do Rio de Janeiro foi seguido Alves et al. (2000).

Para a elaboração dos mapas foram consideradas as principais localidades de registro em território fluminense de cada espécie, citadas na literatura ornitológica (Apêndice I). As coordenadas geográficas das localidades foram obtidas de PAYNTER JR. \& TRAYLOR (1991), VAnzolini (1992) e mapas de 1:50.000 do IBGE (Instituto Brasileiro de Geografia e Estatística). Dados referentes à precipitação e temperatura médias anuais foram obtidos do Sistema de Meteorologia do Estado do Rio de Janeiro (SIMERJ - www.simerj.com) e do Instituto Nacional de Meteorologia (INMET - www. inmet.gov.br), respectivamente.

\section{RESULTADOS E DISCUSSÃO}

Foram encontradas 59 espécies de aves com seu limite norte ou sul de distribuição geográfica localizado no estado do Rio de Janeiro, correspondendo a cerca de
$7,8 \%$ das 749 espécies pertencentes à avifauna conhecida do estado (Gagliardi, 2011). Dessas 59 espécies, 40 (68\%) apresentam limite sul e 19 (32\%) limite norte de distribuição no estado (Tab. I).

Espécies florestais foram predominantes (79,5\%; $\mathrm{N}=47$ ) entre aquelas com limite de distribuição no Rio de Janeiro, sendo que aquelas restritas às matas de baixada e submontanas (até $500 \mathrm{~m}$ de altitude, segundo Veloso et al., 1991) representaram mais da metade $(68 \% ; \mathrm{N}=40)$ das espécies (Fig. 1). Endemismos da Mata Atlântica corresponderam a pouco mais de um terço $(42,5 \%$; $\mathrm{N}=25$ ) das espécies (Tab. II).

Considerando-se as espécies ameaçadas de extinção no estado do Rio de Janeiro, 28 espécies com limite de distribuição no estado (47,5\%) são consideradas ameaçadas (Fig. 2). Sabendo-se que as regiões próximas às margens da distribuição das espécies tendem a exibir densidades populacionais menores (BROwN \& LOMOLINO, 2006), não é surpreendente que quase metade das espécies com limites de distribuição no Rio de Janeiro seja considerada rara e ameaçada de extinção no estado.

A substituição geográfica latitudinal de espécies próximas parece não ser um fator importante na limitação da distribuição das aves na região do estado do Rio de Janeiro, como já constatado ao longo da planície litorânea entre o sul-fluminense e o norte-paulista (BuzzetTi, 2000). Entretanto, Myrmeciza loricata (Lichtenstein, 1823) (ao norte) e Myrmeciza squamosa Pelzeln, 1868 (ao sul) se substituem geograficamente, com pequena sobreposição no extremo sul do litoral fluminense (Francisco Mallet-Rodrigues, obs. pess.). Neopelma aurifrons (Wied, 1831) (ao norte) e Neopelma chrysolophum Pinto, 1944 (ao sul), ainda que segregados altitudinalmente, com a primeira em matas de baixada e a segunda em matas montanas, apresentam um certo padrão de substituição geográfica, com $N$. chrysolophum limitando sua distribuição ao norte do estado do Rio de Janeiro na região do Parque Estadual do Desengano, enquanto $N$. aurifrons tem seu registro mais meridional a cerca de 120 quilômetros a sudoeste, na baixada da região central do estado, em Cachoeiras de Macacu (WhitNey et al., 1995).

Espécies com limite sul de distribuição no estado do Rio de Janeiro. O conjunto de espécies com limite sul de distribuição têm registros em quase todo o território fluminense, com exceção da região do alto vale do rio Paraíba do Sul (incluindo o Maciço do Itatiaia) e a porção mais ocidental da costa fluminense, próximo à divisa com o estado de São Paulo (Figs 3-5). Diversas espécies [Crypturellus soui (Hermann, 1783), Pyrrhura cruentata (Wied, 1820), Pyrrhura leucotis (Kuhl, 1820), Touit surdus (Kuhl, 1820), Amazona rhodocorytha (Salvadori, 1890), Myrmotherula axillaris (Vieillot, 1817), Thamnophilus ambiguus Swainson, 1825, Mionectes oleagineus (Lichtenstein, 1823), Rhynchocyclus olivaceus (Temminck, 1820), Myiarchus tuberculifer (d'Orbigny \& Lafresnaye, 1837), 
Tab. I. Espécies de aves com limite sul ou norte de distribuição geogrática no estado do Rio de Janeiro (A, endêmico da Mata Atlântica; B, mata de baixada; C, mata montana; D, restinga aberta; E, ambientes abertos aquáticos; F, ameaçado de extinção no estado do Rio de Janeiro; G, visitante meridional). Os números representam as principais localidades de registro (Vide Apêndice).

\begin{tabular}{|c|c|c|}
\hline Espécie & Limite sul & Limite norte \\
\hline Crypturellus soui B & $17,21,30,32,45$ & \\
\hline Crypturellus variegatus $\mathrm{B}, \mathrm{F}$ & 30,31 & \\
\hline Cygnus melancoryphus E, G & & 47 \\
\hline Anas flavirostris $\mathrm{E}, \mathrm{G}$ & & 47 \\
\hline Anas versicolor $\mathrm{E}, \mathrm{G}$ & & 47 \\
\hline Netta peposaca E, G & & $20,36,37,44$ \\
\hline Crax blumenbachii A, B, F & 17 & \\
\hline Harpagus bidentatus $\mathrm{B}, \mathrm{C}, \mathrm{F}$ & 17 & \\
\hline Fulica armillata E, G & & $24,39,48$ \\
\hline Fulica rufifrons E, G & & 36 \\
\hline Fulica leucoptera E, G & & 44 \\
\hline Charadrius modestus E, G & & 36,54 \\
\hline Nycticryphes semicollaris E, F & & $20,47,48$ \\
\hline Pyrrhura cruentata A, B, F & $14,17,31,34,42$ & \\
\hline Pyrrhura leucotis A, B, F & $14,25,26,31,43$ & \\
\hline Touit surdus A, B, C, F & $14,25,26,30,31,35,39$ & \\
\hline Amazona rhodocorytha A, B, F & $14,21,31,32,43,51,52$ & \\
\hline Neomorphus geoffroyi B, F & 17 & \\
\hline Phaethornis idaliae A, B, F & $14,30,31,32$ & \\
\hline Discosura langsdorffi $\mathrm{B}, \mathrm{F}$ & $17,25,34,46$ & \\
\hline Discosura longicaudus B & 26 & \\
\hline Monasa morphoeus $\mathrm{B}, \mathrm{F}$ & 39 & \\
\hline Myrmeciza squamosa A, B, C & & $37,40,42,53,54$ \\
\hline Myrmotherula axillaris $\mathrm{B}$ & $4,10,17,30,32,35,38,42,43,45,46,48,49$ & \\
\hline Myrmotherula urosticta A, B, F & 14,31 & \\
\hline Myrmotherula unicolor A, B & & $8,26,30,31,32,35,37,40,42,45,46,52,53$ \\
\hline Formicivora grisea $\mathrm{B}$ & 35 & \\
\hline Formicivora serrana A, C & $1,7,9,11,12,13,15,16,17,18,19$ & \\
\hline Thamnomanes caesius B & $9,14,30,35$ & \\
\hline Dysithamnus xanthopterus A, C & & $22,23,25,27,28,30,35,40$ \\
\hline Dysithamnus plumbeus A, B, F & 2,3 & \\
\hline Thamnophilus ambiguus A, B & $6,28,29,30,32,35,38,42,43,45,46,48,50$ & \\
\hline Cercomacra brasiliana A, B, F & $11,30,36$ & \\
\hline Chamaeza ruficauda A, C & & $22,23,25,27,30,35,40,53$ \\
\hline Dendroplex picus $\mathrm{B}, \mathrm{F}$ & 5 & \\
\hline Phleocryptes melanops E & & 20,44 \\
\hline Heliobletus contaminatus A, C & & $22,23,25,30,35,40$ \\
\hline Thripophaga macroura A, B, C, F & 9,14 & \\
\hline Neopelma aurifrons A, B, F & 30 & \\
\hline Pipra rubrocapilla $\mathrm{B}, \mathrm{F}$ & $14,17,31,32$ & \\
\hline Machaeropterus regulus $\mathrm{A}, \mathrm{B}$ & $14,17,30,31,32$ & \\
\hline Dixiphia pipra $\mathrm{B}, \mathrm{F}$ & $10,17,31,32,38$ & \\
\hline Schiffornis turdina B & $10,30,31,32$ & \\
\hline Tijuca atra A, C & & $14,23,25,27,30,35,40$ \\
\hline Xipholena atropurpurea A, B, F & 14 & \\
\hline Cotinga maculata A, B, F & 10,17 & \\
\hline Piprites pileata A, C, F & & 22,23 \\
\hline Mionectes oleagineus $\mathrm{B}$ & $10,14,26,30,32,35,36,45$ & \\
\hline Rhynchocyclus olivaceus B, F & $3,17,28,30,31,32,42$ & \\
\hline Tolmomyias poliocephalus B & $10,31,32$ & \\
\hline Hemitriccus obsoletus A, C & & $22,23,40$ \\
\hline Ornithion inerme $\mathrm{B}, \mathrm{F}$ & $10,30,32$ & \\
\hline Myiarchus tuberculifer B & $14,30,31,35,43$ & \\
\hline Hymenops perspicillatus $\mathrm{E}, \mathrm{F}, \mathrm{G}$ & & 48 \\
\hline Mimus gilvus $\mathrm{D}, \mathrm{F}$ & $21,47,48,49,51$ & \\
\hline Tangara brasiliensis A, B, F & $9,14,16,30,32,35,43$ & \\
\hline Hemithraupis flavicollis $\mathrm{B}$ & $14,17,30,31,32,33,35,38,39,43,45$ & \\
\hline Caryothraustes canadensis $\mathrm{B}$ & $6,17,30,31,35,45$ & \\
\hline Euphonia xanthogaster B & $10,25,30,31,35,42,45,46$ & \\
\hline
\end{tabular}




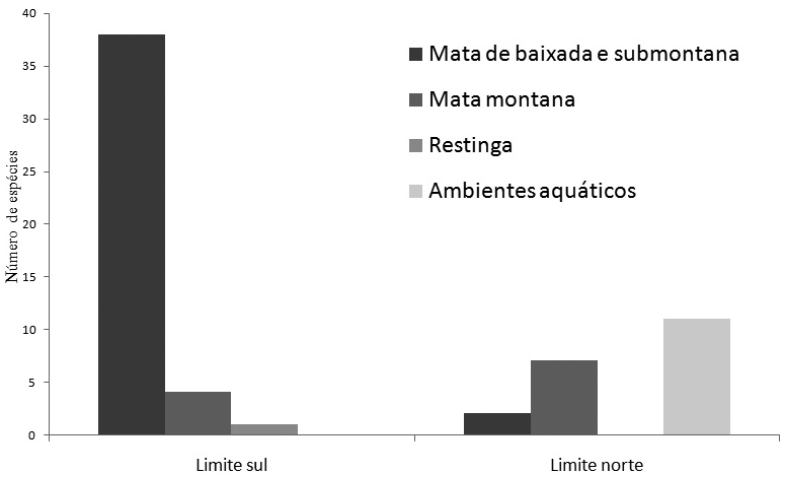

Fig. 1. Número de espécies de aves, com limite sul ou norte de distribuição geográfica no estado do Rio de Janeiro, para cada tipo de habitat básico.

Mimus gilvus (Vieillot, 1807), Tangara brasiliensis (Linnaeus, 1766), Hemithraupis flavicollis (Vieillot, 1818), Caryothraustes canadensis (Linnaeus, 1766) e Euphonia xanthogaster Sundevall, 1834] têm na zona oeste do município do Rio de Janeiro e litoral sul do estado seu limite meridional de distribuição, enquanto outras espécies [Crypturellus variegatus (Gmelin, 1789), Phaethornis idaliae (Bourcier \& Mulsant, 1856), Myrmotherula urosticta (Sclater, 1857), Tolmomyias poliocephalus (Taczanowski, 1884), Ornithion inerme Hartlaub, 1853, Pipra rubrocapilla Temminck, 1821, Machaeropterus regulus (Hahn, 1819), Dixiphia pipra (Linnaeus, 1758), Schiffornis turdina (Wied, 1831)] têm seus registros mais meridionais nas baixadas do norte e centro fluminense. Entretanto, é possível que, no passado, mesmo essas últimas espécies tenham se distribuído até as baixadas mais a oeste, incluindo toda a área compreendendo a atual região metropolitana do Rio de Janeiro, até o município de Mangaratiba. A expressiva proporção $(95 \% ; \mathrm{N}=38)$ de representantes típicos de matas de baixada e submontanas entre as espécies com limite sul de distribuição no estado do

Tab. II. Proporção de espécies de aves florestais (endêmicas e não endêmicas da Mata Atlântica) e não florestais, com limite sul ou norte de distribuição geográfica no estado do Rio de Janeiro.

\begin{tabular}{lcc}
\hline Status & Limite sul & Limite norte \\
\hline $\begin{array}{l}\text { Espécies florestais endêmicas da } \\
\text { Mata Atlântica }\end{array}$ & $17(42,5 \%)$ & $8(42 \%)$ \\
$\begin{array}{l}\text { Espécies florestais não endêmicas } \\
\text { da Mata Atlântica }\end{array}$ & $22(55 \%)$ & 0 \\
Espécies não florestais & $1(2,5 \%)$ & $11(58 \%)$ \\
\hline
\end{tabular}

Rio de Janeiro (Fig. 1) revela a importância desse tipo de ambiente na distribuição dessas espécies de aves na região. Mais da metade $(59,5 \% ; \mathrm{N}=28)$ das localidades com registro dessas espécies situa-se abaixo de $500 \mathrm{~m}$ de altitude (Fig. 3; Tab. III). A barreira física imposta pela Serra do Mar, estreitando a planície costeira em direção ao sul-fluminense, limita localmente as matas de baixada provocando uma descontinuidade avifaunística ao longo do litoral entre o sul do Rio de Janeiro e o norte de São Paulo (Buzzetti, 2000).
Foram predominantes as localidades com precipitação média anual abaixo de $1500 \mathrm{~mm}(74,4 \%$; $\mathrm{N}=35)$ e temperatura média anual acima de $20^{\circ} \mathrm{C}(98 \%$; $\mathrm{N}=46$ ) na distribuição das espécies com limite sul no estado do Rio de Janeiro (Figs 4, 5; Tab. III).

Quase todas as espécies $(97,5 \% ; \mathrm{N}=39)$ habitam ambientes florestais, mas menos da metade $(42,5 \%$; $\mathrm{N}=17$ ) é constituída por endemismos da Mata Atlântica (Tab. II). A proporção de endemismos da Mata Atlântica entre essas espécies, contudo, assemelha-se àquelas obtidas em alguns estudos ornitológicos realizados em território fluminense (SCOTt \& BRooke, 1985; Browne, 2005; Mallet-Rodrigues et al., 2010). Entretanto, a proporção de endemismos da Mata Atlântica entre essas espécies pode aumentar com o reconhecimento como espécies válidas de algumas populações (admitidas como subespécies) restritas ao leste do Brasil.

Mais da metade $(62,5 \% ; \mathrm{N}=25)$ das espécies com seu limite sul de distribuição no Rio de Janeiro está incluída na lista de espécies ameaçadas de extinção do estado (Fig. 2). Algumas dessas espécies [Harpagus bidentatus (Latham, 1790), Neomorphus geoffroyi (Temminck, 1820), Monasa morphoeus (Hahn \& Küster, 1823), Xipholena atropurpurea (Wied, 1820) e Cotinga maculata (Statius Muller, 1776)] não apresentam registros recentes no estado, sendo mesmo consideradas provavelmente extintas no Rio de Janeiro (Alves et al., 2000). Adicionalmente, quatro espécies [Discosura langsdorff (Temminck, 1821), Discosura longicaudus (Gmelin, 1788), Formicivora grisea (Boddaert, 1783) e Thamnomanes caesius (Temminck, 1820)] não incluídas na lista das aves ameaçadas do estado aumentam a proporção de espécies em risco de extinção, dentre aquelas com limite sul no Rio de Janeiro, pois também não têm sido registradas regularmente em território fluminense. A intensa fragmentação das matas de baixada possivelmente é um dos principais fatores para a elevada proporção de espécies ameaçadas entre as aves com limite sul no Rio de Janeiro.

Espécies com limite norte de distribuição no estado do Rio de Janeiro. As espécies com limite norte de distribuição podem ser classificadas em dois

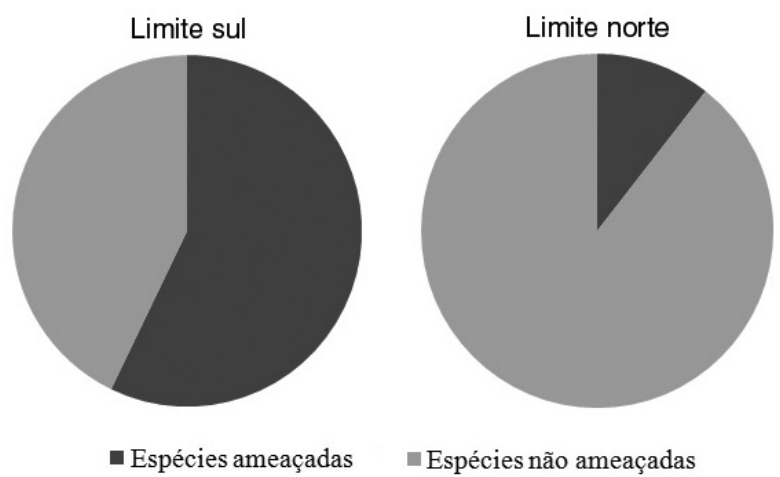

Fig. 2. Proporção de espécies consideradas ameaçadas de extinção entre as aves com limite sul ou norte de distribuição geográfica no estado do Rio de Janeiro. 


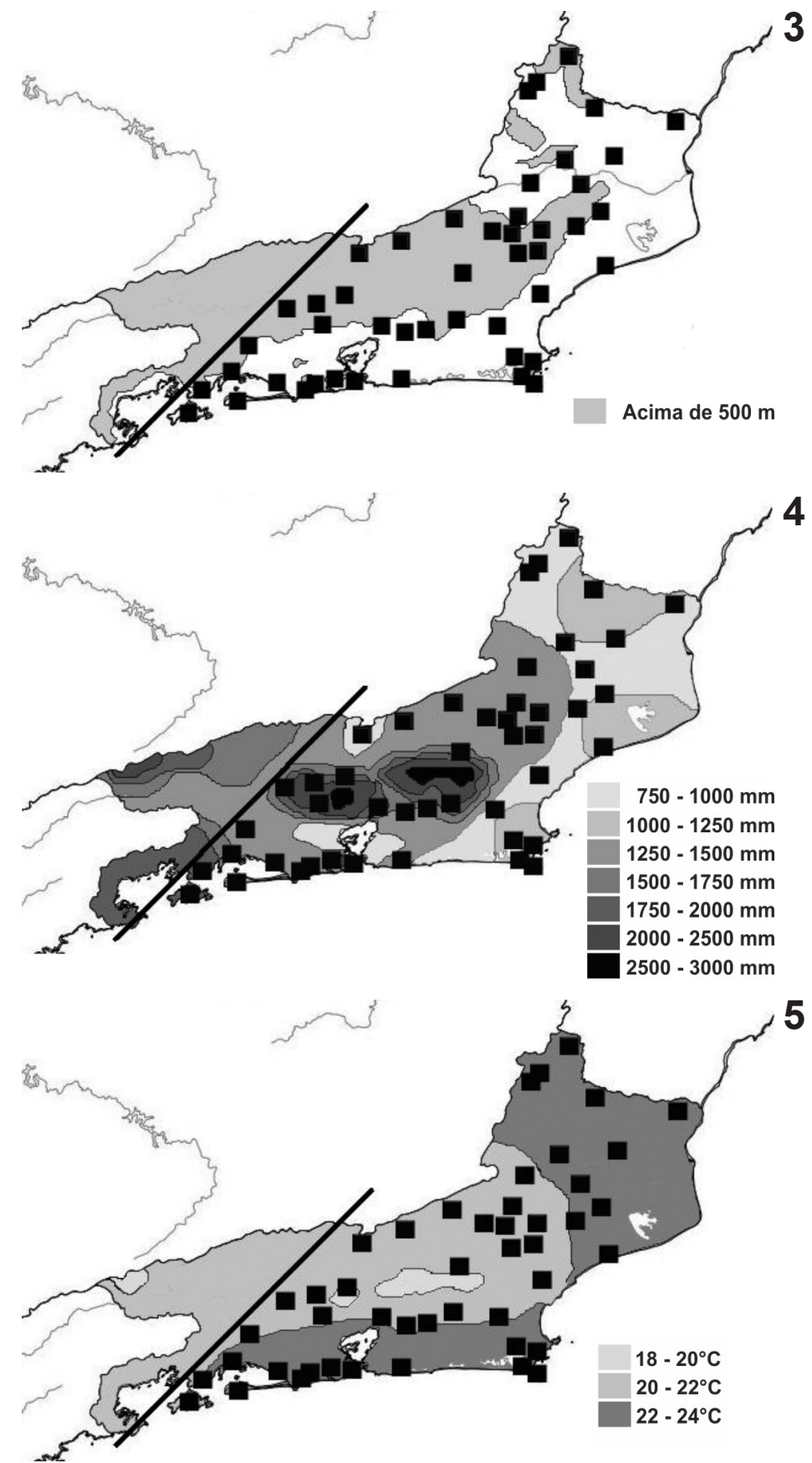

Figs 3-5. Localidades de registro das espécies de aves com limite sul de distribuição geográfica no estado do Rio de Janeiro: 3, altitude; 4, precipitação pluviométrica; 5 , temperatura média anual. Limite sul aproximado da distribuição das espécies limitado pela linha reta.

grupos básicos quanto ao seu padrão de distribuição em território fluminense (Tab. II). O primeiro grupo é composto por espécies de ambientes florestais montanos e endêmicas da Mata Atlântica [Myrmeciza squamosa, Myrmotherula unicolor (Ménétriès, 1835), Dysithamnus xanthopterus Burmeister, 1856, Chamaeza ruficauda (Cabanis \& Heine, 1859), Heliobletus contaminatus Berlepsch, 1885, Tijuca atra Ferrusac, 1829, Piprites pileata (Temminck, 1822) e Hemitriccus obsoletus (Miranda-Ribeiro, 1906)], que se distribuem, principalmente, pelo maciço do Itatiaia e pelas formações montanhosas constituintes da Serra do Mar (serras da Bocaina, do Tinguá e dos Órgãos), tendo a
Serra do Desengano como limite setentrional (Fig. 6). Algumas dessas espécies (Dysithamnus xanthopterus, Chamaeza ruficauda, Heliobletus contaminatus, Tijuca atra, Piprites pileata e Hemitriccus obsoletus) também podem ser encontradas na porção norte do Maciço do Itatiaia e partes da Serra da Mantiqueira, localizadas em território mineiro adjacente à divisa com o Rio de Janeiro (Francisco Mallet-Rodrigues, obs. pess.). Para essas espécies, a depressão do rio Paraíba do Sul não parece representar (ao menos em sua porção média) uma importante barreira geográfica (SILVA \& STRAUBE, 1996). Entretanto, o baixo rio Paraíba do Sul pode representar uma efetiva barreira a distribuição dessas espécies, pois 
nenhuma delas tem registro ao norte da região. Contudo, a presença dessas espécies parece estar bastante relacionada às regiões das matas pluviais montanas do litoral, com precipitação e temperatura anuais médias acima de $1500 \mathrm{~mm}$ e entre 20 e $22^{\circ} \mathrm{C}$, respectivamente (Figs 7, 8; Tab. III), coincidindo com as regiões 1 e 4 (mais chuvosas do estado) de ANDré et al. (2008).

O segundo grupo de espécies [Cygnus melancoryphus (Molina, 1782), Anas flavirostris Vieillot, 1816, Anas versicolor Vieillot, 1816, Netta peposaca (Vieillot, 1816), Fulica armillata Vieillot,
1817, Fulica rufifrons Philippi \& Landbeck, 1861, Fulica leucoptera Vieillot, 1817, Charadrius modestus Lichtenstein, 1823, Nycticryphes semicollaris (Vieillot, 1816), Phleocryptes melanops (Vieillot, 1817), Hymenops perspicillatus (Gmelin, 1789)] é constituído, principalmente, por visitantes ocasionais associados a ambientes aquáticos abertos e costeiros, em áreas com precipitação e temperatura anuais médias abaixo de 1500 mm e acima de $22^{\circ} \mathrm{C}$, respectivamente (Figs 6-8; Tab. III), encontrando na região das lagoas costeiras (Maricá, Saquarema, Araruama e Feia) o seu limite norte de

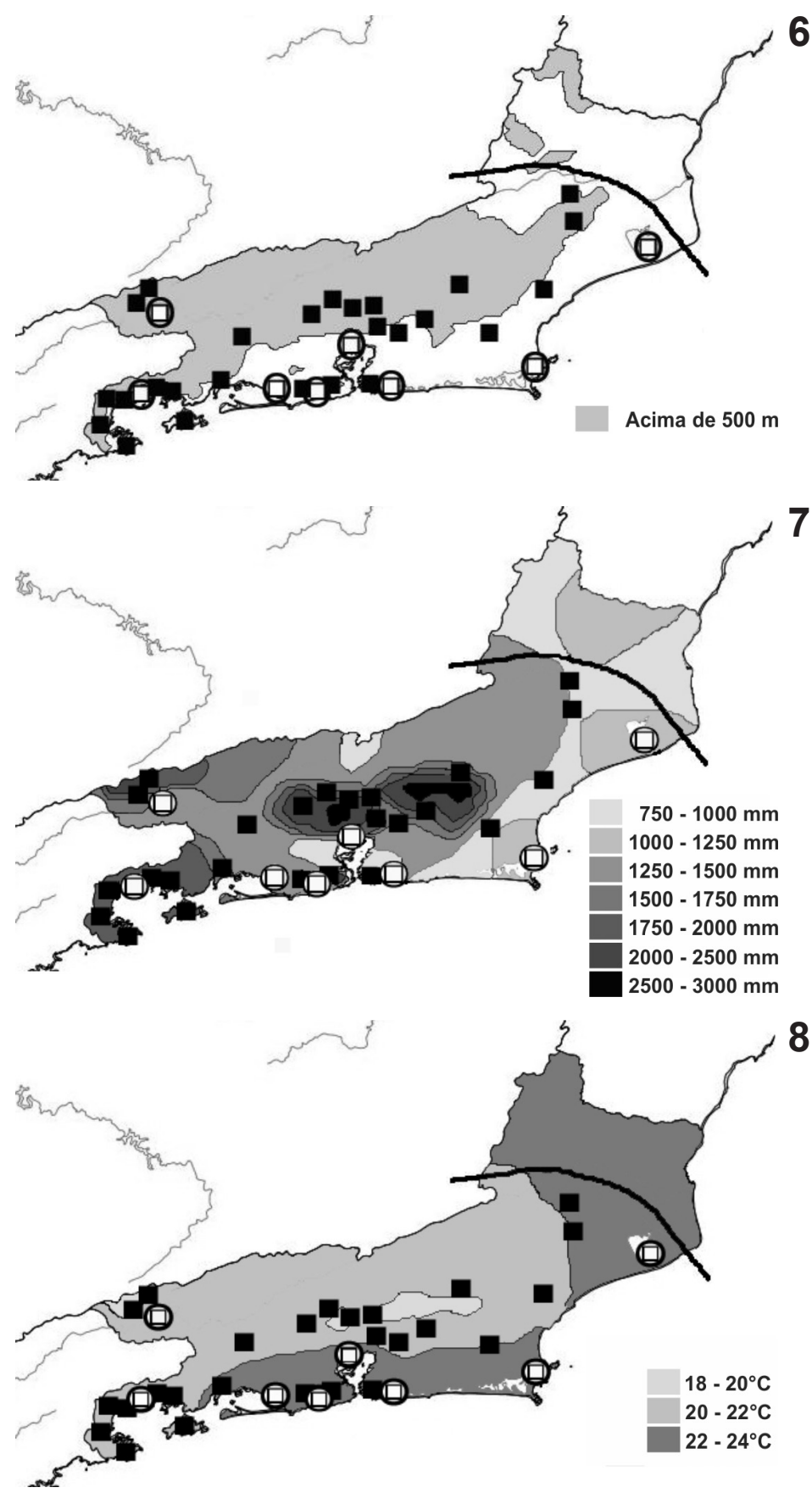

Figs 6-8. Localidades de registro das espécies de aves com limite norte de distribuição geográfica no estado do Rio de Janeiro: 6, altitude; 7, precipitação pluviométrica; 8, temperatura média anual. Quadrados negros representam as localidades de registro das espécies florestais e quadrados brancos dentro de círculos representam as localidades de registro das espécies de ambientes aquáticos. Limite norte aproximado da distribuição das espécies limitado pela linha curva. 
Tab. III. Número (e frequência) de localidades de registro de espécies de aves com limites sul e norte de distribuição no estado do Rio de Janeiro para cada fator climático avaliado.

\begin{tabular}{lccc}
\hline Fatores climáticos & Limite sul & $\begin{array}{c}\text { Limite norte } \\
\text { (espécies florestais) }\end{array}$ & $\begin{array}{c}\text { Limite norte } \\
\text { (espécies aquáticas) }\end{array}$ \\
\hline Altitude $(\mathrm{m})$ & $28(59,5 \%)$ & $9(34,5 \%)$ & $7(87,5 \%)$ \\
Abaixo de 500 & $19(40,5 \%)$ & $17(65,5 \%)$ & $1(12,5 \%)$ \\
Acima de 500 & & & $1(12,5 \%)$ \\
& & & $2(25 \%)$ \\
Precipitação média anual $(\mathrm{mm})$ & $12(25,6 \%)$ & $5(19,2 \%)$ & $4(50 \%)$ \\
$750-1000$ & $6(12,7 \%)$ & 0 & 0 \\
$1000-1250$ & $17(36,2 \%)$ & $3(11,5 \%)$ & $1(12,5 \%)$ \\
$1250-1500$ & $3(6,4 \%)$ & $2(7,6 \%)$ & 0 \\
$1500-1750$ & $6(12,7 \%)$ & $13(50 \%)$ & 0 \\
$1750-2000$ & $3(6,4 \%)$ & $3(11,5 \%)$ & 0 \\
$2000-2500$ & 0 & & 0 \\
$2500-3000$ & & $4(15,3 \%)$ & $2(25 \%)$ \\
Temperatura média anual $\left({ }^{\circ} \mathrm{C}\right)$ & $1(2 \%)$ & $15(57,6 \%)$ & $6(75 \%)$ \\
$18-20$ & $23(49 \%)$ & $7(26,9 \%)$ & \\
$20-22$ & $23(49 \%)$ & & \\
$22-24$ & & & \\
\hline
\end{tabular}

distribuição. Algumas dessas espécies (Anas flavirostris, Anas versicolor e Phleocryptes melanops) distribuem-se também pela Cordilheira dos Andes e costa pacífica da América do Sul alcançando regiões mais setentrionais (SicK, 1997).

As aves de ambientes aquáticos representam pouco mais da metade $(58 \% ; \mathrm{N}=11)$ das espécies com limite norte de distribuição no Rio de Janeiro (Fig. 1), sendo que a maioria $(82 \% ; \mathrm{N}=9)$ dessas espécies aquáticas é constituída por visitantes ocasionais de inverno em seus deslocamentos sazonais para o norte de sua área de distribuição. Somente Nycticryphes semicollaris e Phleocryptes melanops são residentes no Rio de Janeiro. Fulica armillata têm sido registrada também na primavera e verão na região dos lagos fluminenses (Francisco Mallet-Rodrigues, obs. pess.).

Somente duas espécies (10,5\%) com limite norte de distribuição no Rio de Janeiro são consideradas ameaçadas no estado (Fig. 2). Uma dessas espécies é endêmica da Mata Atlântica, encontrada em matas montanas acima de 1000 m (Piprites pileata), e a outra uma discreta espécie de planícies alagadas (Nycticryphes semicollaris). Porém, ainda que não consideradas ameaçadas no estado, todas as demais espécies de ambientes aquáticos com limite norte de distribuição têm sido raramente ou apenas muito localmente registradas na região.

A baixa representatividade de táxons ameaçados entre as espécies com limite norte de distribuição no estado, pode ser explicada pela elevada proporção $(87,5 \% ; N=7)$ de representantes de matas montanas entre suas espécies florestais, pois as matas das regiões montanhosas têm sido melhor preservadas que as de áreas de baixada, no leste do Brasil (Hueck, 1972; Rizzini, 1997). Consequentemente, as maiores concentrações de espécies ameaçadas têm sido encontradas nas áreas de menor altitude (Buzzetti, 2000; Mallet-Rodrigues et al., 2010). No entanto, duas importantes áreas para as espécies de ambientes aquáticos citadas nesse estudo encontramse em preocupante situação de ameaça. A região dos lagos fluminenses (entre os municípios de Maricá e Cabo Frio) sofre uma intensa degradação ambiental decorrente do aumento populacional e turismo de massa, ampliado com a inauguração da ponte Rio-Niterói, em 1973, e mais recentemente pela intensificação da exploração de petróleo na Bacia de Campos. No sul fluminense, a lagoa da Turfeira (ou lagoa da Kodak), localizada as margens do rio Paraíba do Sul, em Resende, e que constitui-se em um refúgio importante para diversas espécies de aves aquáticas, encontra-se em constante ameaça de assoreamento e aterro pelo projeto de ampliação do Pólo Industrial de Resende. Ambas as áreas devem ser consideradas prioritárias para a conservação ambiental.

Compreender os padrões de distribuição das espécies em seus limites geográficos extremos, bem como seus fatores limitantes, é de extrema importância não somente na determinação dos limites faunísticos regionais, mas também por possuir implicações no estabelecimento de modelos ecológicos, como os de distribuição potencial, e nos esforços conservacionistas.

Agradecimentos. Sou grato a Gloria D.A. Castiglioni pelas valiosas críticas e sugestões a uma versão anterior desse trabalho.

\section{REFERÊNCIAS BIBLIOGRÁFICAS}

Aleixo, A. \& Vielliard, J. M. E. 1995. Composição e dinâmica da avifauna da Mata de Santa Genebra, Campinas, São Paulo, Brasil. Revista Brasileira de Zoologia 12:493-511.

Alves, M. A. S. \& VeCCH, M. B. 2009. Birds, Ilha Grande, state of Rio de Janeiro, Southeastern Brazil. Check List 5(2):300-313. Disponível em $<$ http://www.checklist.org.br/getpdf?SL005-09>. Acesso em: 13.04.2012. 
Alves, M. A. S.; Pacheco, J. F.; Gonzaga, L. A. P.; Cavalcanti, R. B.; Raposo, M. A.; Yamashita, C.; Maciel, N. C. \& Castanheira, M. 2000. Aves. In: Bergallo, H. G.; Rocha, C. F. D.; Alves, M. A. S. \& Van Sluys, M. orgs. A fauna ameaçada de extinção do Estado do Rio de Janeiro. Rio de Janeiro, EdUERJ. p. 113-124.

André, R. G. B.; Marques, V. da S.; Pinheiro, F. M. A. \& Ferraudo, A. S. 2008. Identificação de regiões pluviometricamente homogêneas no estado do Rio de Janeiro, utilizando-se valores mensais. Revista Brasileira de Meteorologia 23(4):501-509.

Barth, R. 1957. A fauna do Parque Nacional do Itatiaia. Boletim do Parque Nacional de Itatiaia 6:1-150.

Batalha Filho, H; Amaral, F. R. do \& Firme, D. H. 2011. New specimens extend the range of Serra Antwren Formicivora serrana. Cotinga 33:96-97.

Bauer, C. \& Pacheco, J. F. 2000. Lista das aves da região de Visconde de Mauá, Serra da Mantiqueira, no limite dos Estados do Rio de Janeiro e Minas Gerais. Atualidades Ornitológicas 97:7.

Bencke, G. A.; Maurício, G. N.; Develey, P. F. \& Goerck, J. M. orgs. 2006. Áreas Importantes para a Conservação das Aves no Brasil. Parte I - Estados do Domínio da Mata Atlântica. São Paulo, SAVE Brasil.

Brown, J. H. \& Lomolino, M. V. 2006. Biogeografia. 2 ed. Ribeirão Preto, FUNPEC Editora. 691p.

Browne, P. W. P. 2005. The birds of Parati, south-east Brazil. Cotinga 24:85-98.

Burmeister, H. 1855-1856. Systematische Üebersicht der Thiere Brasiliens welche während einer Reise durch die Provinzen von Rio de Janeiro und Minas Geraës gesammelt oder beobachtet wurden von Dr. Hermann Burmeister, ö Prof. d. Zoologie und Direct. d. Zool. Mus. der Universität zu Halle. Vols. 2 e 3 (Vögel). Berlin, Georg Reimer.

Buzzetti, D. R. C. 2000. Distribuição altitudinal de aves em Angra dos Reis e Parati, sul do Estado do Rio de Janeiro, Brasil. In: Alves, M. A. S.; Silva, J. M. C. da; Van Sluys, M.; Bergallo, H. DE G. \& Rocha, C. F. D. DA. orgs. A ornitologia no Brasil: Pesquisa atual e perspectivas. Rio de Janeiro, EdUERJ. p.131-148.

Cabanis, J. 1874. Uebersicht der von Herrn Carl Euler im District Cantagallo, Provinz Rio de Janeiro, gesammelten Vögel. Journal für Ornithologie 22(125):81-90; 22(126):225-231.

Castro-Astor, I.; Alves, M. A. S. \& Cavalcanti, R. B. 2004 Display behavior and spatial distribution of the Red-headed Manakin in the Atlantic Forest of Brazil. Condor 106(2):320 335

2007. Display behavior and spatial distribution of the White-crowned Manakin in the Atlantic Forest of Brazil Condor 109(1): 155-166.

CBRO - Comitê Brasileiro de Registros Ornitológicos. 2011. Listas das aves do Brasil. 10 ed. Disponível em $<$ http://www. cbro.org.br>. Acesso em: 10.03.2012.

Coelmo, E. P.; Alves, V. S.; Fernandez, F. A. S. \& Soneghet, M. L. L. 1991. On the bird faunas of coastal islands of Rio de Janeiro state, Brazil. Ararajuba 2:31-40.

Collar, N. J.; Gonzaga, L. P.; Krabbe, N.; Madroño Nieto, A.; Naranjo, L. G.; Parker III, T. A. \& Wege, D. C. 1992. Threatened birds of the America: The ICBP/IUCN Red Data Book. Cambridge, ICBP. 1150p.

Costa, L. P.; Leite, Y. L. R.; Fonseca, G. A. B. Da \& Fonseca, M. T. DA. 2000. Biogeography of South American Forest Mammals: Endemism and Diversity in the Atlantic Forest. Biotropica 32(4):872-881.

Cracraft, J. 1985. Historical biogeography and patterns of differentiation within the South America avifauna: areas of endemism. In: Buckley, P. A.; Foster, M. S. \& Morton, E. S. orgs. Neotropical Ornithology. Washington, American Ornithologists Union (Ornithological Monograhs 36). p.4984.

Cunha, A. A. \& RajÃo, H. 2007. Mamíferos terrestres e aves da Terra Indígena Sapukai (Aldeia Guarani do Bracui [sic]), Angra dos Reis, RJ, Brasil. Boletim do Museu de Biologia Mello Leitão (n. sér.) 21:19-34.
Dean, W. 1996. A ferro e fogo: a história e devastação da Mata Atlântica brasileira. São Paulo, Companhia das Letras. 484p.

Descourtilz, J. T. 1854-1856. Ornithologie Brésilienne ou Histoire des Oiseaux du Bresil, remarquables par leur plumage, leur chant ou leurs habitudes. Rio de Janeiro, Thomas Reeves. $42 \mathrm{p}$.

Euler, C. 1867. Beiträge zur Naturgeschichte der Vögel Brasiliens. Journal für Ornithologie 15(87):177-198; 15(88):217-233, 399-420

Ferreira, I.; GouvÊA, M. T. J. \& Ueda, M. M. 1986. Levantamento preliminar da avifauna da Estação Ecológica de Piraí, Estado do Rio de Janeiro. Arquivos da Universidade Federal Rural do Rio de Janeiro:45-53.

Firme, D. H. \& Raposo, M. A. 2011. Taxonomy and geographic variation of Formicivora serrana (Hellmayr, 1929) and Formicivora littoralis Gonzaga \& Pacheco, 1990 (Aves: Passeriformes: Thamnophilidae). Zootaxa 2742:1-33.

Gagliardi, R. 2011. Avifauna completa do Estado do Rio de Janeiro. Táxeus - Listas de espécies. Disponível em $<$ http:// www.taxeus.com.br/lista.jsf?c=82>. Acesso em: 11.02.2012.

Gomes, V. S. M.; Loiselle, B. A. \& Alves, M. A. S. 2008a. Birds foraging for fruits and insects in shrubby restinga vegetation, southeastern Brazil. Biota Neotropica 8:21-31.

Gomes, V. S. M.; Correia, M. C. R.; Lima, H. A. \& Alves, M. A. S. 2008b. Potential role of frugivorous birds (Passeriformes) on seed dispersal of six plant species in a restinga habitat, southeastern Brazil. Revista de Biologia Tropical 56(1):205216.

Gonzaga, L. P. \& Pacheco, J. F. 1990. Two new subspecies of Formicivora serrana (Hellmayr) from southeastern Brazil, and notes on the type locality of Formicivora deluzae Ménétriés. Bulletin of the British Ornithological Club 110(4):187-193.

Guimarães, M. A. 2001a. Levantamento da avifauna do complexo lagunar de Araruama, Rio de Janeiro, Brasil. Atualidades Ornitológicas 101:12.

2001b. Levantamento da avifauna do complexo lagunar de Araruama, Rio de Janeiro, Brasil - Parte 2. Atualidades Ornitológicas 102:12.

Guimarães, M. A. \& Guimarães, P. L. C. 2006. Levantamento preliminar da avifauna da Serra de Sapiatiba, Região dos Lagos, Rio de Janeiro, Brasil. Atualidades Ornitológicas 133 (Online). Disponível em <http://www.ao.com.br/download sapiatib.pdf>. Acesso em 25.07.2011.

Hellmayr, C. E. 1929. Catalogue of birds of the Americas. Field Museum of Natural History, Zoological Series 13(6):1-258 (Publ. 266).

Hueck, K. 1972. As florestas da América do Sul: ecologia, composição e importância econômica. São Paulo, Polígono. $466 \mathrm{p}$.

Ihering, H. von. 1900. Aves observadas em Cantagallo e Nova Friburgo. Revista do Museu Paulista 4:149-164.

Krabbe, N. 2007. Birds collected by P.W. Lund and J.T. Reinhardt in south-eastern Brazil between 1825 and 1855, with notes on P.W. Lund's travels in Rio de Janeiro. Revista Brasileira de Ornitologia 15(3):331-357.

MAcArthuR, R. H. 1972. Geographical ecology: patterns in the distributions of species. New York, Harper \& Row. 269p.

Maciel, E. 2009. Aves do Município do Rio de Janeiro. Rio de Janeiro, Technical Books. 407p.

Mallet-Rodrigues, F. \& Noronha, M. L. M. DE. 2003. The avifauna of low elevations in the Serra dos Órgãos, Rio de Janeiro state, south-east Brazil. Cotinga 20:51-56.

2009. Birds in the Parque Estadual dos Três Picos, Rio de Janeiro state, south-east Brazil. Cotinga 31:96-107.

Mallet-Rodrigues, F.; Parrini, R. \& Pacheco, J. F. 2007. Birds of the Serra dos Órgãos, State of Rio de Janeiro, Southeastern Brazil: a review. Revista Brasileira de Ornitologia 15(1):535

Mallet-Rodrigues, F.; Parrini, R.; Pimentel, L. M. S. \& Bessa, R. 2010. Altitudinal distribution of birds in a mountainous region in southeastern Brazil. Zoologia 27(4):503-522.

Mallet-Rodrigues, F.; Alves, V. S.; Noronha, M. L. M.; Serpa, G. A.; Soares, A. B. A.; Couto, G. S.; Maciel, E.; Madeira, S. \& 
Draghi, J. 2008. Aves da Baixada de Jacarepaguá, Município do Rio de Janeiro, Estado do Rio de Janeiro. Revista Brasileira de Ornitologia 16(3):221-231.

Pacheco, J. F.; Astor, I. N. C. \& Cesar, C. B. 2010. Avifauna da Reserva Biológica de Poço das Antas, Silva Jardim, RJ. Atualidades Ornitológicas 157 (Online):55-74. <Disponível em http://www.ao.com.br/download/AO157 55.pdf $>$. Acesso em: 04.05.2011.

Pacheco, J. F. \& Bauer, C. 1997. Novo limite sul na distribuição do arapaçu-de-bico-branco Xiphorhynchus picus no leste do Brasil. Atualidades Ornitológicas 78:13.

Pacheco, J. F. \& Gonzaga, L. P. 1994. Tiranídeos do Estado do Rio de Janeiro provenientes de regiões austrais da América do Sul. Nótulas Faunísticas 63:1-4.

Pacheco, J. F. \& Parrini, R. 1998a. Registros questionáveis de aves do Estado do Rio de Janeiro. I - Non-Passeres. Atualidades Ornitológicas 81:5.

1998b Registros questionáveis de aves do Estado do Rio de Janeiro. II - Passeres. Atualidades Ornitológicas 83:6-7.

Pacheco, J. F.; Parrini, R; Fonseca, P. S. M.; Whitney, B. M. \& Maciel, N. C. 1996. Novos registros de aves para o Estado do Rio de Janeiro: Região Norte. Atualidades Ornitológicas 72:10-12.

Pacheco, J. F.; Parrini, R.; Whitney, B. M.; Bauer, C. \& Fonseca, P. S. M. 1997a. Novos registros de aves para o estado do Rio de Janeiro: Costa Verde. Atualidades Ornitológicas 78:4-5.

1997b. Novos registros de aves para o estado do Rio de Janeiro: região sul do vale do rio Paraíba do Sul. Atualidades Ornitológicas 79:4-5.

Parker, T. A. \& Goerck, J. M. 1997. The importance of national parks and biological reserves to bird conservation in the Atlantic Forest of Brazil. Ornithological Monographs 48:527-541.

Parker, T. A.; Stotz, D. F. \& Fitzpatrick, J. W. 1996. Ecological and distributional databases. In: Stotz, D. F.; FitzPatrick, J. W.; Parker, T. A. \& Moskovits, D. K. eds. Neotropical birds: ecology and conservation. Chicago, University of Chicago Press. p. 113-436.

Paynter JR., R. A. \& Traylor, M. A. 1991. Ornithological Gazetteer of Brazil. 2 vols. Cambridge, Museum of Comparative Zoology.

Pelzeln, A. von. 1868-1871. Zur Ornithologie Brasiliens. Resultate von Johann Natterer Reisen in den Jahren 1817 1835. 3 vols. Wien, Pichler's Witwe \& Sohn.

Pimentel, L. \& Olmos, F. 2011. The birds of Reserva Ecológica Guapiaçu (REGUA), Rio de Janeiro, Brazil. Cotinga 33:8-24.

Pinto, O. M. O. 1938. Catálogo das aves do Brasil e lista dos exemplares que as representam no Museu Paulista. Revista do Museu Paulista 22(1937):1-566.

1944. Catálogo das aves do Brasil e lista dos exemplares existentes na coleção do Departamento de Zoologia. 2 Parte. São Paulo, Sec. Agric. Ind. e Comércio. 700p.

1945. Cinqüenta anos de investigação ornitológica. Arquivos de Zoologia 4:261-340.

1951. Aves do Itatiaia - Lista remissiva e novas achegas à avifauna da região. Papéis Avulsos do Departamento de Zoologia 10:155-208.

1954. Aves do Itatiaia. Boletim do Parque Nacional de Itatiaia 3:1-87.

1978. Novo catálogo das aves do Brasil. Primeira Parte: Aves não Passeriformes e Passeriformes não Oscines, com exclusão da família Tyrannidae. São Paulo, Empresa Gráfica da Revista dos Tribunais. 446p.

Piratelli, A.; Sousa, S. D.; Corrêa, J. S.; Andrade, V. A.; Ribeiro, R. Y.; Avelar, L. H. \& Oliveira, E. F. 2008. Searching for bioindicators of forest fragmentation: passerine birds in the Atlantic forest of southeastern Brazil. Brazilian Journal of Biology 68(2):259-268.

Rizzini, C. T. 1997. Tratado de Fitogeografia do Brasil: aspectos ecológicos, sociológicos e florísticos. Rio de Janeiro, Âmbito Cultural Edições. 747p

Rocha, C. F. D.; Bergallo, H. G.; Van Sluys, M.; Alves, M. A. S. \& JAMEL, C. E. 2007. The remnants of restinga habitats in the brazilian Atlantic Forest of Rio de Janeiro state, Brazil: Habitat loss and risk of disappearance. Brazilian Journal of Biology 67(2):263-273.

SClater, P. L. 1886. Catalogue of the Passeriformes or perching birds in the collection of the British Museum. Fringilliformes. Part 2. Coerebidae, Tanagridae and Icteridae. Catalogue of the Birds in the British Museum. Vol. 11. London, British Museum (Natural History).

1888. Catalogue of the Passeriformes or perching birds in the collection of the British Museum. Oligomyodae. Tyrannidae, Oxyrhamphidae, Pipridae, Cotingidae, Phytotomidae, Philepittidae, Pittidae, Xenicidae and Eurylaemidae. Catalogue of the Birds in the British Museum. Vol. 14 London, British Museum (Natural History).

Scott, D. A. \& Brooke, M. DE L. 1985. The endangered avifauna of Southeastern Brazil: a report on the BOU/WWF expeditions of 1980/81 and 1981/82. In: Diamond, A. W. \& Lovejoy, T. E. eds. Conservation of Tropical Forest Birds. Cambridge, ICBP (Techn. Publ. 4). p. 115-139.

Sick, H. 1962. Die Buntschnepfe, Nycticryphes semicollaris in Brasilien. Journal für Ornithologie 103(1):102-107.

1997. Ornitologia Brasileira. Rio de Janeiro, Nova Fronteira.

Sigrist, M. S. \& Carvalho, C. J. B. 2008. Detection of areas of endemism on two spatial scales using Parsimony Analysis of Endemicity (PAE): the Neotropical region and the Atlantic Forest. Biota Neotropical 8(4). Disponível em $<$ http:// www.biotaneotropica.org.br/v $8 \mathrm{n} 4 / \mathrm{en} / \mathrm{abstract}$ ?article+ bn00308042008>. Acesso em: 27.5.2011.

Silva, J. M. C.; Sousa, M. C. \& Castelletti, C. H. M. 2004. Areas of endemism for passerine birds in the Atlantic forest, South America. Global Ecology and Biogeography 13(1):85-92.

Silva, J. M. C. \& Straube, F. C. 1996. Systematics and biogeography of Scaled Woodcreepers (Aves: Dendrocolaptidae). Studies Neotropical Fauna and Environment 31:3-10

Silveira, L. F. \& Uezu, A. 2011. Checklist das aves do Estado de São Paulo, Brasil. Biota Neotropical 11(1a). Disponível em $<$ http://www.biotaneotropica.org.br/v11n $1 \mathrm{a} / \mathrm{pt} /$ abstract?inven tory+bn0061101a2011>. Acesso em: 09.02.2012.

Simpson, R. \& Simpson, E. 2011. Registro documentado da batuírade-peito-tijolo Charadrius modestus (Charadriiformes: Charadriidae) em Paraty, Rio de Janeiro. Atualidades Ornitológicas 162:18-19.

Terborgh, J. 1971. Distribution on environmental gradients: theory and a preliminary interpretation of distributional patterns in the avifauna of the Cordillera Vilcabamba, Peru. Ecology 52(1):23-40.

Terborgh, J. \& Weske, J. S. 1975. The role of competition in the distribution of Andean birds. Ecology 56(3):562-576.

Vanzolini, P. E. 1992. A supplement to the Ornithological Gazetteer of Brazil. São Paulo, Museu de Zoologia da Universidade de São Paulo. 252p.

Veloso, H. P.; Rangel Filho, A. L. R. \& Lima, J. C. A. 1991. Classificação da vegetação brasileira, adaptada a um sistema universal. Rio de Janeiro, IBGE (Departamento de Recursos Naturais e Estudos Ambientais). 124p.

Ventura, P. E. C. \& Ferreira, I. 2009. Avifauna da Mata Atlântica do Estado do Rio de Janeiro. Rio de Janeiro Technical Books. 256p.

Whitney, B. M.; Pacheco, J. F. \& Parrini, R. 1995. Two species of Neopelma in southeastern Brazil and diversification within the Neopelma/Tyranneutes complex: implications of the subspecies concept for conservation (Passeriformes: Tyrannidae). Ararajuba 3:43-53.

Willis, E. O. 1979. The composition of avian communities in remanescent woodlots in southern Brazil. Papéis Avulsos de Zoologia 33:1-25.

Willis, E. O. \& Oniki, Y. 2003. Aves do Estado de São Paulo. Rio Claro, Editora Divisa. 532p.

Zimmer, K. \& Isler, M. 2003. Family Thamnophilidae (typical antbirds). In: Del Hoyo, J; Elliott, A. \& Christie, D. eds. Handbook of the Birds of the World. Vol. VIII. Broadbills to Tapaculos. Barcelona, Lynx Ediciones. p. 448-681. 
Apêndice I. Principais localidades de registro das espécies de aves consideradas nesse estudo e suas fontes bibliográficas.

1. Varre-Sai $\left(20^{\circ} 55^{\prime} \mathrm{S}, 41^{\circ} 52^{\prime} \mathrm{W}\right)($ FIRME \& RAPOSO, 2011)

2. Natividade $\left(21^{\circ} 02^{\prime} \mathrm{S}, 41^{\circ} 58^{\prime} \mathrm{W}\right)$ (Collar et al., 1992)

3. Raposo, Itaperuna ( $\left.21^{\circ} 06^{\prime} \mathrm{S}, 41^{\circ} 06^{\prime} \mathrm{W}\right)$ (Collar et al., 1992; PACHECo et al., 1996)

4. Bom Jesus do Itabapoana $\left(21^{\circ} 08^{\prime} \mathrm{S}, 41^{\circ} 41^{\prime} \mathrm{W}\right)$ (PACHECO et al., 1996)

5. São Francisco de Itabapoana (ca. $\left.21^{\circ} 19^{\prime} \mathrm{S}, 41^{\circ} 01^{\prime} \mathrm{W}\right)$ (PACHECo \& BAUER, 1997)

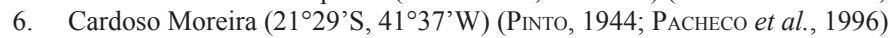

7. Cambuci (21 $\left.34^{\prime} \mathrm{S}, 41^{\circ} 54^{\prime} \mathrm{W}\right)$ (FIRME \& RAPOSO, 2011)

8. São Fidélis $\left(21^{\circ} 39^{\prime} \mathrm{S}, 41^{\circ} 44^{\prime} \mathrm{W}\right)$ (KRABBE, 2007)

9. Itaocara $\left(21^{\circ} 41^{\prime} \mathrm{S}, 42^{\circ} 04^{\prime} \mathrm{W}\right)$ (KRABBE, 2007)

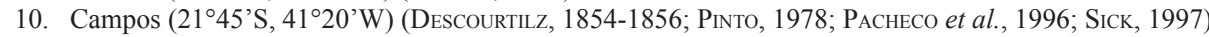

11. Carmo (21 $\left.{ }^{\circ} 56^{\prime} \mathrm{S}, 42^{\circ} 36^{\prime} \mathrm{W}\right)$ (Batalha Filho et al., 2011; Firme \& Raposo, 2011)

12. Sapucaia ( $\left.21^{\circ} 59^{\prime} \mathrm{S}, 42^{\circ} 54^{\prime} \mathrm{W}\right)$ (Batalha Filho et al., 2011; Firme \& Raposo, 2011)

13. Três Rios $\left(22^{\circ} 07^{\prime}\right.$ 'S, $\left.43^{\circ} 12^{\prime} \mathrm{W}\right)$ (Batalha Filho et al., 2011; Firme \& RAPOso, 2011)

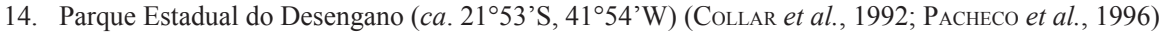

15. São Sebastião do Alto ( $\left.21^{\circ} 57^{\prime} \mathrm{S}, 42^{\circ} 08^{\prime} \mathrm{W}\right)$ (Batalha Filho et al., 2011; Firme \& Raposo, 2011)

16. Santa Maria Madalena (21 $57^{\prime}$ S, $42^{\circ} 00^{\prime} \mathrm{W}$ ) (Pacheco et al., 1996; Batalha Filho et al., 2011; Firme \& Raposo, 2011)

17. Cantagalo ( $\left.21^{\circ} 58^{\prime} \mathrm{S}, 42^{\circ} 22^{\prime} \mathrm{W}\right)$ (Euler, 1867; CABANIS, 1874; IHERING, 1900)

18. Macuco (2 $\left.1^{\circ} 59^{\prime} \mathrm{S}, 42^{\circ} 15^{\prime} \mathrm{W}\right)$ (Batalha Filho et al., 2011; Firme \& Raposo, 2011)

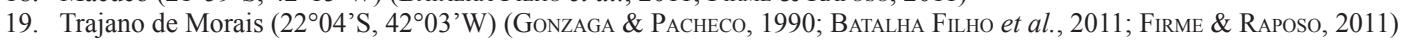

20. Lagoa Feia $\left(22^{\circ} 00^{\prime} \mathrm{S}, 41^{\circ} 20^{\prime} \mathrm{W}\right)$ (PINTO, 1945; SICK, 1962, 1997)

21. Parque Nacional da Restinga de Jurubatiba (ca. $22^{\circ} 17^{\prime}$ S, $41^{\circ} 41^{\prime} \mathrm{W}$ ) (Rocha et al., 2007; Gomes et al., 2008a,b)

22. Visconde de Mauá, Resende (22²0’S, $\left.44^{\circ} 33^{\prime} \mathrm{W}\right)$ (BAUER \& PACHECO, 2000)

23. Parque Nacional de Itatiaia (ca. 22²9’S, 44³3’W) (Pinto, 1944, 1951, 1954; Barth, 1957; Collar et al., 1992; Parker \& Goerck, 1997; SICK, 1997)

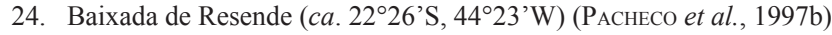

25. Nova Friburgo ( $22^{\circ} 16^{\prime}$ 'S, $42^{\circ} 32^{\prime}$ 'W) (Burmeister, 1855-1856; Sclater, 1886, 1888; Scott \& Brooke, 1985, Mallet-Rodrigues \& Noronha, 2009)

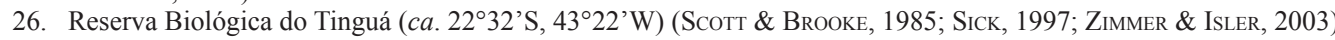

27. Petrópolis $\left(22^{\circ} 31^{\prime} \mathrm{S}, 43^{\circ} 10^{\prime} \mathrm{W}\right)$ (Hellmayr, 1929)

28. Miguel Pereira ( $\left.22^{\circ} 27^{\prime} \mathrm{S}, 43^{\circ} 28^{\prime} \mathrm{W}\right)$ (Piratelli et al., 2008)

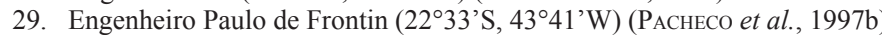

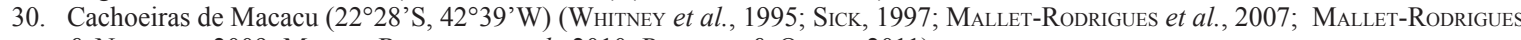
\& Noronha, 2009; Mallet-Rodrigues et al., 2010; Pimentel \& Olmos, 2011)

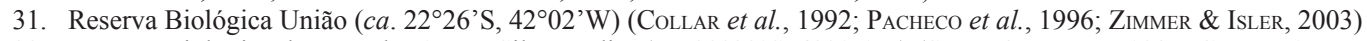

32. Reserva Biológica de Poço das Antas, Silva Jardim (ca. 22³3'S, 42¹7’W) (SCOTt \& Brooke, 1985; CAStro-Astor et al., 2004, 2007; PACHECo et al., 2010)

33. Silva Jardim (22 $\left.39^{\prime} \mathrm{S}, 42^{\circ} 23^{\prime} \mathrm{W}\right)$ (MALlet-Rodrigues \& Noronha, 2009)

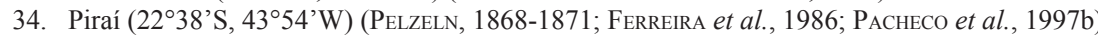

35. Teresópolis $\left(22^{\circ} 24^{\prime} \mathrm{S}, 42^{\circ} 57^{\prime} \mathrm{W}\right)$ e Guapimirim (22 $\left.32^{\circ} \mathrm{S}, 42^{\circ} 59^{\prime} \mathrm{W}\right)$ (SCOTt \& Brooke, 1985; Mallet-Rodrigues \& Noronha, 2003; Mallet-Rodrigues et al., 2007, 2010)

36. Magé (22³9’S, $\left.43^{\circ} 02^{\prime} \mathrm{W}\right)$ (Sick, 1997)

37. Angra dos Reis $\left(23^{\circ} 00^{\prime} \mathrm{S}, 4^{\circ} 18^{\prime} \mathrm{W}\right)$ (PACheco et al., 1997a; Buzzetti, 2000; Cunha \& RajÃo, 2007)

38. Serra de Sapiatiba, São Pedro da Aldeia e Iguaba Grande (22 $49^{\prime}$ S, $\left.42^{\circ} 09^{\prime} \mathrm{W}\right)$ (GuIMARÃES \& GuIMARÃEs, 2006)

39. Cabo Frio (22 $2^{\circ} 3^{\prime}$ S, $42^{\circ} 01^{\prime}$ W) (Pelzeln, 1868-1871; Sick, 1997)

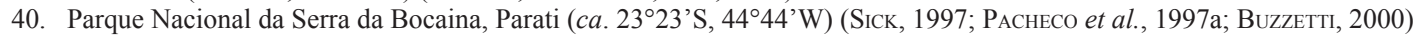

41. Mambucaba, Angra dos Reis $\left(23^{\circ} 01^{\prime} \mathrm{S}, 44^{\circ} 32^{\prime} \mathrm{W}\right)$ (BuzzetTi, 2000)

42. Mangaratiba (22 $\left.57^{\prime} \mathrm{S}, 4^{\circ} 02^{\prime} \mathrm{W}\right)$ (Pelzeln, 1868-1871; Coelho et al., 1991)

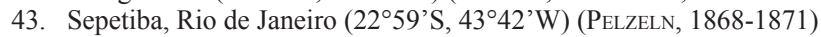

44. Guaratiba, Rio de Janeiro (2300'S, $\left.43^{\circ} 39^{\prime} \mathrm{W}\right)$ (MACIEL, 2009)

45. Parque Estadual da Pedra Branca, Rio de Janeiro (ca. $2^{\circ} 56^{\prime} \mathrm{S}, 43^{\circ} 28^{\prime} \mathrm{W}$ ) (Ventura \& Ferreira, 2009; MAciel, 2009)

46. Parque Nacional da Tijuca, Rio de Janeiro ( $c a .22^{\circ} 57^{\prime} \mathrm{S}, 43^{\circ} 17^{\prime} \mathrm{W}$ ) (PARKer \& GoercK, 1997; MACIEL, 2009)

47. Maricá (2256’S, 4250’W) (Sick, 1997; Rocha et al., 2007)

48. Baixada de Jacarepaguá, Rio de Janeiro (ca. 22 $2^{\circ} 58^{\prime} \mathrm{S}, 43^{\circ} 22^{\prime} \mathrm{W}$ ) (MAllet-Rodrigues et al., 2008; MACIEL, 2009; Pacheco \& GonZaga, 1994)

49. Restinga de Massambaba, Arraial do Cabo (ca. 22 $\left.{ }^{\circ} 56^{\prime} \mathrm{S}, 42^{\circ} 12^{\prime} \mathrm{W}\right)$ (Guimarães, 2001a,b)

50. Ilha de Cabo Frio, Arraial do Cabo (22 $\left.2^{\circ} 9^{\prime} \mathrm{S}, 41^{\circ} 59^{\prime} \mathrm{W}\right)$ (Coelho et al., 1991)

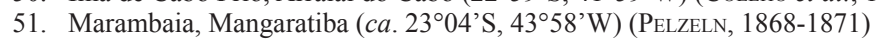

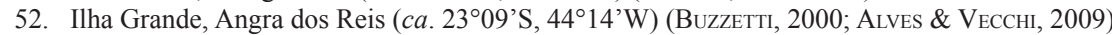

53. Parati ( $\left.c a .23^{\circ} 13^{\prime} \mathrm{S}, 4^{\circ} 43^{\prime} \mathrm{W}\right)$ (Buzzetti, 2000; Browne, 2005; Simpson \& Simpson, 2011)

54. Área de Proteção Ambiental do Cairuçu, Parati (23ำ $\left.18^{\prime} \mathrm{S}, 4^{\circ} 35^{\prime} \mathrm{W}\right)$ (Buzzetti, 2000)

Recebido em 26 de julho de 2012. Aceito 13 de outubro de 2012. ISSN 0073-4721

Artigo disponível em: www.scielo.br/isz 